\title{
Neuroradiologic Features in X-linked $\alpha$-Thalassemia/Mental Retardation Syndrome
}

\author{
T. Wada, H. Ban, M. Matsufuji, N. Okamoto, K. Enomoto, K. Kurosawa, and N. Aida
}

\begin{abstract}
BACKGROUND AND PURPOSE: X-linked $\alpha$-thalassemia/mental retardation syndrome (Mendelian Inheritance in Man, 301040) is one of the X-linked intellectual disability syndromes caused by mutations of the ATRX gene and characterized by male predominance, central hypotonic facies, severe cognitive dysfunction, hemoglobin $\mathrm{H}$ disease ( $\alpha$-thalassemia), genital and skeletal abnormalities, and autistic and peculiar behavior. More than 200 patients in the world, including >70 Japanese patients, have been diagnosed with ATR-X syndrome.
\end{abstract}

MATERIALS AND METHODS: We reviewed the brain MRI and/or CT findings of 27 Japanese patients with ATR-X with ATRX mutations retrospectively.

RESULTS: The findings were categorized into 5 types: 1) nonspecific brain atrophy (17/27); 2) white matter abnormalities, especially around the trigones (11/27); 3) widespread and scattered white matter abnormalities (1/27); 4) delayed myelination (4/27); and 5) severe and rapidly progressive cortical brain atrophy (1/27).

CONCLUSIONS: This is the first report on a comprehensive study of brain MRI/CT findings of ATR-X syndrome. Our findings suggest that the ATRX protein seems to be involved in normal myelination. The classification will require revisions in the near future, but it will be helpful in establishing the relationship between ATRX mutation and brain development and understanding the ATRX protein function in the brain.

ABBREVIATIONS: ADD domain $=$ ATRX-DNMT3-DNMT3L; ATR-X $(A T R X)=X$-linked $\alpha$-thalassemia $/$ mental retardation

$\mathbf{X}$ -linked $\alpha$-thalassemia/mental retardation syndrome (Mendelian Inheritance in Man, 301040) is one of the X-linked intellectual disability syndromes and is due to mutations of the ATRX gene on Xq13.3, encoding a SWI/SNF-like chromatin remodeling protein. ${ }^{1}$ The ATRX protein has 2 functionally important domains: the zinc-finger motif (ADD) and the highly conserved chromatin-remodeling domain, where the ATRX mu-

Received September 28, 2012; accepted after revision December 17

From the Divisions of Neurology (T.W.), Genetics (K.E., K.K.), and Radiology (N.A.), Kanagawa Children's Medical Center, Yokohama, Japan; Department of Pediatrics (H.B.), Himeji Red Cross Hospital, Himeji, Japan; Department of Pediatrics (M.M.),

Yanagawa Institute for Developmental Disabilities, Yanagawa, Japan; and Department of Molecular Medicine (N.O.), Osaka Medical Center and Research Institute for Maternal and Child Health, Osaka, Japan.

This work was supported by a research grant for Intractable Diseases from the Ministry of Health, Labour and Welfare of Japan (H22-nanchi-ippan-114).

Paper previously presented in the poster session at: American Society of Human Genetics hosting the 12th International Congress of Human Genetics, October 11-15, 2011; Montreal, Quebec, Canada.

Please address correspondence to Takahito Wada, MD, PhD, 2-138-4 Mutsukawa, Minami-ku, Yokohama, Kanagawa 232-8555, Japan; e-mail: takahito.wada0001@me.com

- Indicates open access to non-subscribers at www.ajnr.org

= Indicates article with supplemental on-line table

http://dx.doi.org/10.3174/ajnr.A3560 tations cluster. ${ }^{2}$ Although the pathophysiologic mechanism of ATR-X syndrome is not yet completely known, ATR-X syndrome is one of the chromatin diseases (which include Rett, CoffinLowry, and Rubinstein-Taybi syndromes), and a disturbance of the epigenetic mechanism is suggested. ${ }^{3}$

More than 200 patients in the world, $>70$ of whom are Japanese, have been diagnosed with ATR-X syndrome. ${ }^{2,4}$ ATR-X syndrome seems to be a rare disease; however, we estimate that the prevalence of ATR-X syndrome is 1/30,000-40,000 neonate boys, which is much higher than previously reported estimations (K. Kurosawa, unpublished data 2012). Therefore, more patients remain to be diagnosed because 7-9 patients with ATR-X should be diagnosed every year based on the fact we have 1 million neonates born every year in Japan.

In 2010, we established the ATR-X Syndrome Network Japan (http://kcmc.jp/ATR-X/index.html) for patients and their families, and we have surveyed patients with ATR-X syndrome in Japan. The ultimate purpose of our clinical research is to establish diagnostic criteria, which would facilitate further clinical study of individuals with molecularly proved ATR-X, assist in the evaluation of those who appear to exhibit the clinical features of ATR-X with no ATRX mutations, and establish the management of ATR-X syndrome. 

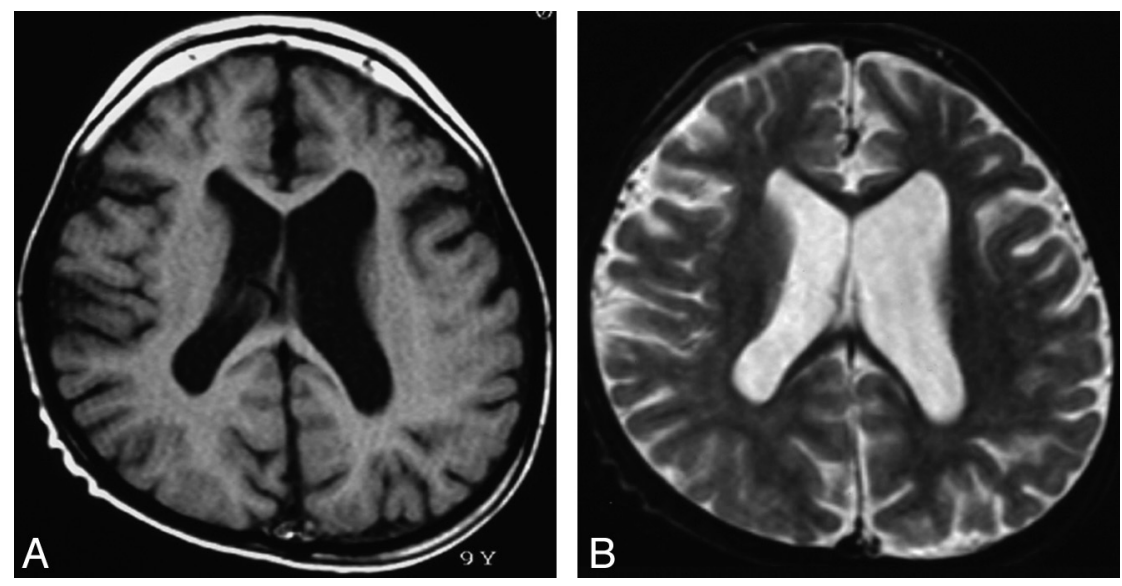

FIG 1. Type 1. Nonspecific brain atrophy. TIWI (A) and T2WI (B) of a 9-year-old patient (case 4) with an ATRX mutation of the ADD domain in exon 6 (c.390 391 ins A; El31fs). Nonprogressive diffuse cortical brain atrophy and ventricular enlargement due to loss of white matter volume are shown.
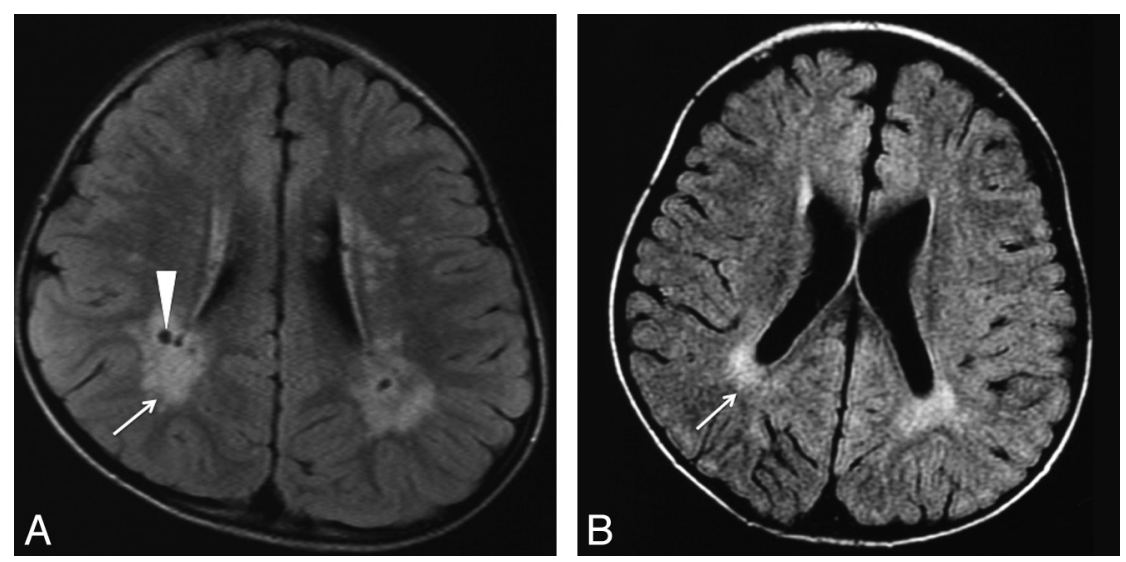

FIG 2. Type 2. White matter abnormalities, especially around the trigones. FLAIR image of a 4-year-old patient (case 22) (A) with an ATRX mutation of the chromatin-remodeling domain in exon 19 (p.V1624M), and a 35-month-old patient (case 21) (B) with an ATRX mutation of the chromatin-remodeling domain in exon 19 (p.A1622V). Increased signal intensity on TIWI/FLAIR in the periventricular region, especially around the peritrigonal area (arrow), and enlargement of perivascular space (arrowhead) are seen.
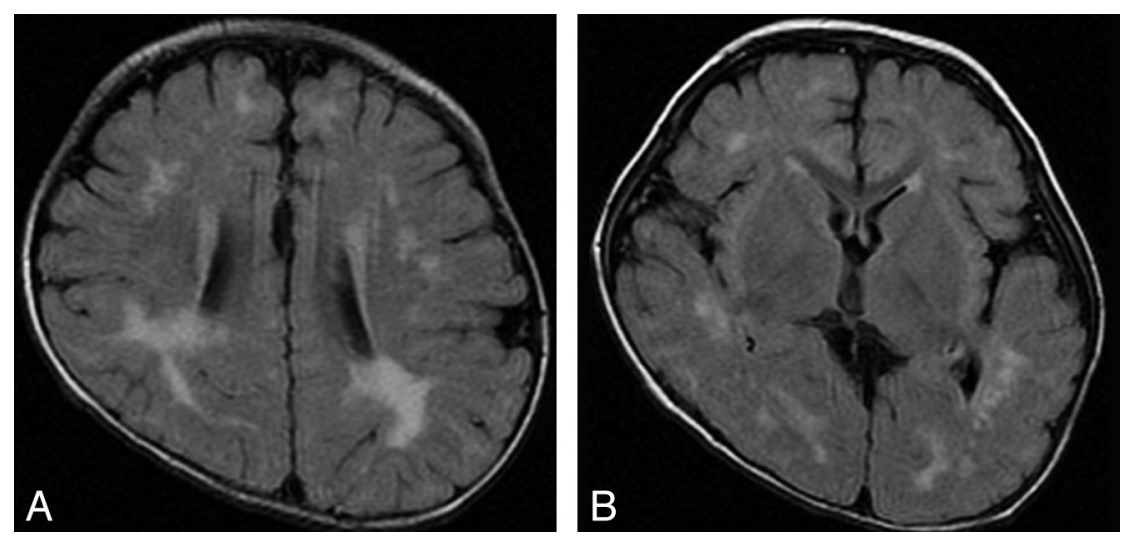

FIG 3. Type 3. Widespread and scattered white matter abnormalities. FLAIR image of a 12month-old patient (case 1) with an ATRX mutation of a nucleotide substitution in $5^{\prime}$-UTR. Note high signal intensity on FLAIR/T2WI in the white matter, especially in the peritrigonal area and deep white matter, not in a diffuse but in a widespread and scattered pattern.

Because few comprehensive studies of brain MRI/CT of ATR-X syndrome are available, we propose here the classification of brain MRI/CT findings in the case of ATR-X syndrome.

\section{MATERIALS AND METHODS}

We reviewed the brain MRI/CT findings of 27 Japanese patients with ATR-X who exhibited ATRX mutations retrospectively. The images came from our medical center and from referrals sent to our center for consultation and spanned 19942012. They were evaluated by a pediatric neuroradiologist, a pediatric radiologist, or pediatric neurologists. The mutations of patients $5-18$ reside in the ADD domain; those of patients 20-24, in the chromatin-remodeling domain. Other mutations are outside these 2 domains, and all of these mutations cause loss-offunction mutations (On-line Table). Their ages at the time of the evaluated MRI/CT ranged from 4 months to 54 years of age. MR imaging of the brain was performed at $1.5 \mathrm{~T}$ or $3 \mathrm{~T}$ at multiple medical centers. Axial T1WI, T2WI, and FLAIR or CT scans were evaluated. DWI was examined in a limited number of patients. Patients with severe prematurity and clinical signs of neonatal hypoxic-ischemic injury that would result in white matter and/or cortex abnormalities on MRI or CT scans were excluded from this study.

\section{RESULTS}

The MRI/CT findings and ATRX mutations of 27 patients with ATR-X are summarized in the On-line Table. The brain CT scans/MRI of all 27 patients whose scans were available showed some abnormal findings: Nonspecific brain atrophy (type 1, Fig 1) was shown in 17 of 27 patients (63\%); a high intensity of white matter especially around the trigones, or the terminal zone, with/without multiple small spheric foci on FLAIR/T2WI (type 2, Fig 2) was seen in 11 patients (41\%). Type 2 with hypoplasia of the cerebellar vermis was shown in 1 patient (case 22), and type 2 with hypoplasia of the corpus callosum and ventricular enlargement was seen in 1 patient (case 25). Compared with type 1, type 2 seemed more frequent in patients with mutations in the chromatin-remodeling domain. Four had hypoplasia of the corpus callosum. Furthermore, widespread and scattered white matter abnormalities on FLAIR (type 3, Fig 3 ) were seen in 1 patient (case 1). Delayed myelination (type 4, Fig 4) was shown in $4(15 \%)$, and severe and rapidly progressive cortical brain atrophy with ventricular enlargement (type 5, Fig 5) was seen in 1 patient (case 27). No alterations of cerebral diffusion 
could be observed on DWI in any patients with types 3, 4, and 5 .

\section{DISCUSSION}

This is the first report on a comprehensive study of brain CT/MRI findings of patients with ATR-X syndrome, though some case reports or personal accounts mentioned some findings of brain MRI/CT, including mild cerebral atrophy, partial or complete agenesis of the corpus callosum, and hypoplasia of the white matter. ${ }^{5,6}$ Our results suggest that brain MRI/CT can present a broader spectrum of abnormalities in both white and gray matter than has been expected in ATR-X syndrome. Some of our patients with ATR-X were referred to our medical center because their MRI/CT scans showed white matter abnormalities, or myelination; and for some, leukodystrophy was suspected as their diagnosis. Therefore, we categorized the MRI/CT findings of 27 patients with ATR-X into 5 types from the point of view of white matter lesions, or myelination.

More patients showed nonspecific brain atrophy (type 1) on brain MRI/CT. This result agrees with the finding that, in a review of 168 patients with ATR-X syndrome, 77\% presented with microcephaly. ${ }^{7}$ This brain atrophy in ATR-X syndrome is not pro-
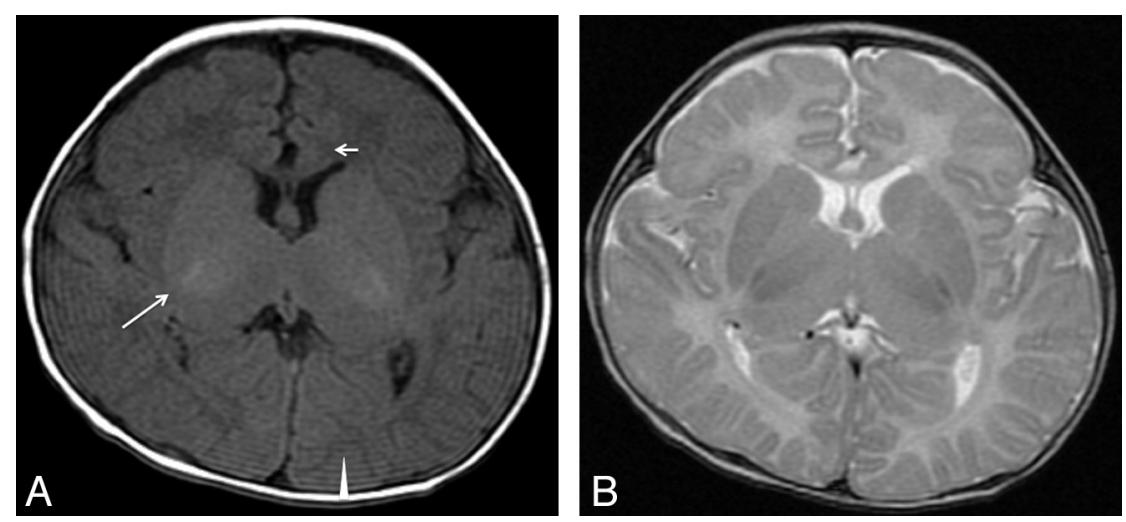

FIG 4. Type 4. Delayed myelination. TIWI (A) and T2WI (B) of a 4-month-old patient (case 26) with an ATRX mutation in exon 35 (c.7156C > T, p.Arg2386Stop). Myelination appears only at the posterior limb of the internal capsule (long arrow) on TIWI. At 4 months of age in a healthy infant, high intensity should extend from the junction of the anterior limb of the internal capsule at the callosal genu (short arrow) all the way back to the visual cortex (arrowhead) along the internal capsule and optic radiations.
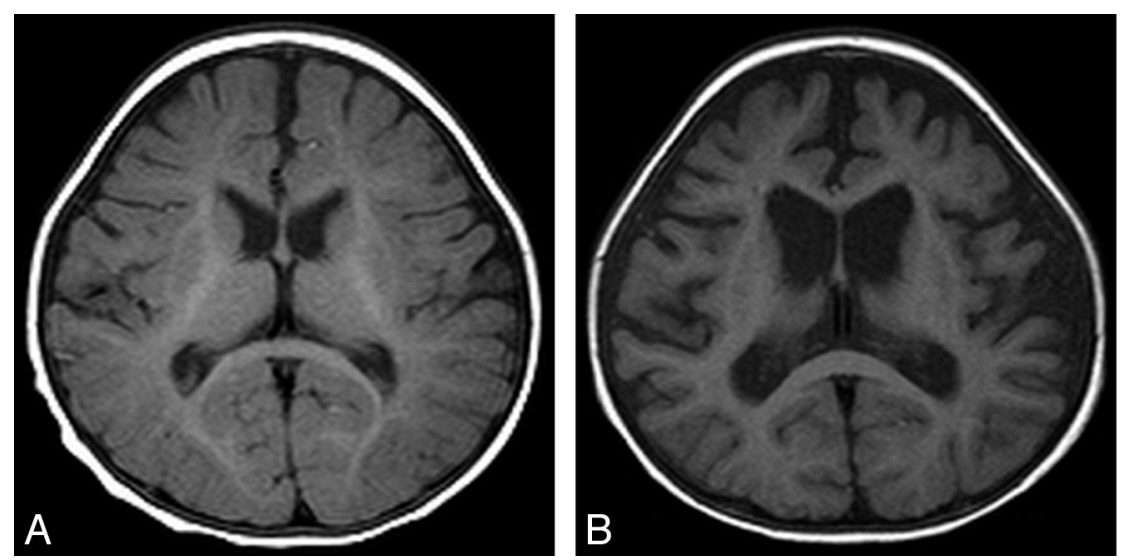

gressive, and it seems to be due to the reduced production of neurons or glia in the perinatal period, not to some destructive process. This characterization was supported by a study by Bérubé et $\mathrm{al}^{8}$ by using a conditional targeting approach with mice, showing that $A T R X$ is a critical mediator of cell survival during early neuronal differentiation and that increased neuronal loss may contribute to severe mental retardation. ${ }^{8}$

Types 2 and 3 suggest an abnormality of the white matter, or myelination. Type 2 includes white matter abnormalities, especially around the trigones, with increased signal intensity showing on T2WI/FLAIR in the periventricular region. Persistently high signal intensity in this peritrigonal area, or the terminal zones, is seen throughout the first decade of life, and it is sometimes very difficult to differentiate from white matter injury resulting from prematurity, which results in periventricular leukomalacia. Moreover, a layer of myelinated white matter is present between the trigones of the ventricle and the terminal zones in healthy patients. ${ }^{9}$ The findings shown in our patients with ATR-X differ from slow myelination in these areas or terminal zones, being normal variants in their distribution and signal intensity. Because these patients did not present clinically with apparent neonatal asphyxia and their findings do not show other signs suggesting periventricular leukomalacia, these white matter abnormalities may be relatively frequent findings in CT/MRI of ATR-X syndrome.

Especially type 3, with its widespread and scattered white matter abnormalities, strongly suggests that normal expression of the ATRX protein is involved in normal myelination because the patient's mutation is a nucleotide substitution in $5^{\prime}$ UTR and ATRX expression should be reduced, though the ATRX protein structure is normal. Actually, red cells of case 1 had more hemoglobin $\mathrm{H}$ inclusions (5\%) than those of other patients, and he had hypospadias. Most interesting, his development was much better than that of typical patients with ATR-X, and he can walk and speak a few words. This suggests that the quantity of the ATRX pro-

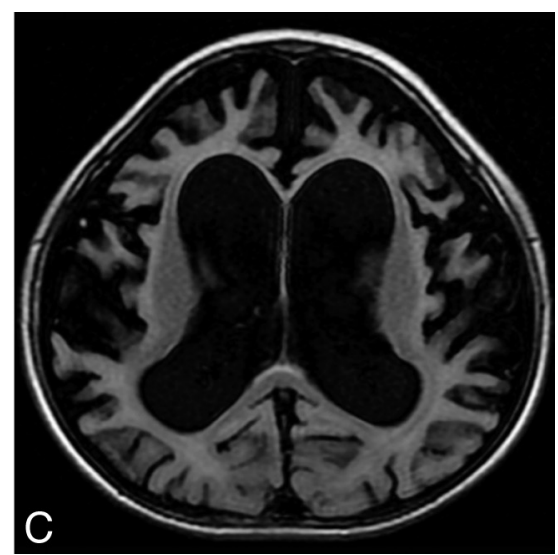

FIG 5. Type 5. Progressive brain atrophy. Sequential change of brain MR imaging of patient 27 with an ATRX mutation in int 35 (c.7200 $+4 A>G$, p.L240Ifs) at 6 months (TIWI) (A), 14 months (TIWI) (B), and 34 months (FLAIR) (C). 
tein can affect $\alpha$-globin expression, genital development, and myelination, but the quality of the ATRX protein is more important for intellectual ability. Another possibility is that the site of the mutation may be more important for ATRX expression in the oligodendroglia, which is involved in myelination. The delayed myelination in type 4 also supports the idea that ATRX is important for normal myelination.

High signal on T2WI/FLAIR and no alteration on DWI in the white matter of these patients meaning that there is no cytotoxic edema but increased water content in the white matter region, and these findings may suggest the prematurity of the blood-brain barrier in ATR-X syndrome, though our data have limitations in that not all MRI/CT scans include diffusion imaging or contrast enhancement.

Most interesting, 2 patients with ATR-X with partial duplications of the ATRX gene, resulting a severe reduction of ATRX messenger RNA and absence of the ATRX protein, presented with normal brain MR imaging findings and agenesis of the corpus callosum, respectively. ${ }^{10}$ The former patient was evaluated before his death at 4.5 months of age, and it was difficult to detect white matter abnormalities. For the latter patient, although the age when the MR imaging was evaluated was 6 months, we cannot specify his age. He could have been too young for evaluation of myelination on MR imaging, and agenesis of the corpus callosum is the ultimate result of white matter abnormality. That finding does not contradict our theory that $A T R X$ is important for normal myelination. However, more data should be forthcoming to clarify the relation between the ATRX protein and white matter or myelination.

Progressive brain atrophy in type 5 is an exceptional finding of CT/MRI in ATR-X syndrome. The patient with type 5 presented with a severe developmental delay and intractable epilepsy following West syndrome, which is rare in ATR-X syndrome. He had no episodes of hypoxic encephalopathy. Although it is possible that he may have had some other pathologic conditions, ATR-X syndrome should be considered in the differential diagnosis of patients with progressive brain atrophy on brain MRI/CT. His mutation was in int 35 , or the last intron, resulting in skipping exon 35 , which consists of 126 base pairs, and the introduction of a 43-amino-acid-deleted ATRX protein. This truncated protein may be related to his severe intellectual disability or epileptic condition.

A gray matter abnormality seems more frequent in those patients with ATRX mutation in the ADD domain, or exons 8, 9, and 10 , and a white matter abnormality in the chromatin-remodeling domain, or exons 18 to 31 . These results would indicate that mutations in the ADD domain produce more severe and permanent psychomotor deficiencies than those in chromatin-remodeling domains, though the clear phenotype-genotype correlation remains to be established. ${ }^{11}$ However, conclusion may be too hasty because we have studied fewer cases with ATRX mutations in the chromatin-remodeling domains.

A number of intellectual disorders have been identified whose gene products regulate chromatin and chromosome architecture, and ATR-X syndrome is a disease of chromatin remodeling, as well as Rett syndrome ( $\mathrm{MeCP} 2)$ and Cornelia de Lange syndrome (SMC1A, SMC3, and NIPBL). ${ }^{3,12}$ These 3 diseases share clinical manifestations, including severe intellectual disabilities, and ATRX, MeCP2, and cohesion proteins, respectively, indicating that these syndromes directly or indirectly interact with each other. Although these syndromes share a common final pathway to their pathogenesis, there are no common characteristic findings on brain MRI/CT among these diseases, except for brain hypoplasia or atrophy. These abnormal signals in the white matter on MR imaging seem to be relatively specific findings in ATR-X syndrome.

We propose that these findings should be included as associated $(<50 \%)$ or supporting features of the diagnostic criteria for ATR-X syndrome. We will evaluate the brain MR imaging/CT of additional patients to validate these findings and update the consensus for the neuroradiologic features as a diagnostic criteria of ATR-X syndrome in the near future.

\section{CONCLUSIONS}

This is the first report on a comprehensive study of brain MRI/CT findings of ATR-X syndrome. We consider ATR-X syndrome a differential diagnosis in patients with intellectual disabilities whose brain MRI shows abnormal signals in the white matter. The ATRX protein seems to be involved in normal myelination. The classification will require revisions in the near future but will be helpful to establish the relationship between the ATRX gene mutation and brain development and to understand the ATRX protein function in the brain.

\section{ACKNOWLEDGMENTS}

We thank our patients and their families, their doctors, and the ATR-X Research Group in Japan: H. Osaka of the Kanagawa Children's Medical Center, Yokohama, Japan; S. Saitoh of the Nagoya City University Graduate School of Medical Science, Nagoya, Japan; N. Okamoto of the Osaka Medical Center and Research Institute for Maternal and Child Health, Osaka, Japan; Y. Fukushima of Shinshu University, Matsumoto, Japan; N. Matsumoto of Yokohama City University, Yokohama, Japan; H. Tanabe of The Graduate University for Advanced Studies, Hayama, Japan; and Y. Goto of the National Center of Neurology and Psychiatry, Kodaira, Japan. We appreciate Dr. Masahide Goto and Dr. Yoshiro Saito (National Center of Neurology and Psychiatry, Kodaira, Japan) for their useful information.

Disclosures: Takahito Wada—RELATED: Grant: This work is supported by a research grant for Intractable Diseases from the Ministry of Health, Labour and Welfare of Japan (H22-nanchi-ippan-114).

\section{REFERENCES}

1. Gibbons RJ, Picketts DJ, Villard L, et al. Mutations in a putative global transcriptional regulator cause $\mathrm{X}$-linked mental retardation with alpha-thalassemia (ATR-X syndrome). Cell 1995;80:837-45

2. Gibbons RJ, Wada T, Fisher CA, et al. Mutations in the chromatinassociated protein ATRX. Hum Mutat 2008;29:796-802

3. Tsankova N, Renthal W, Kumar A, et al. Epigenetic regulation in psychiatric disorders. Nat Rev Neurosci 2007;8:355-67

4. Wada T, Kubota T, Fukushima Y, et al. Molecular genetic study of Japanese patients with $\mathrm{X}$-linked alpha-thalassemia/mental retardation syndrome (ATR-X). Am J Med Genet 2000;94:242-48

5. Wada T, Nakamura M, Matsushita Y, et al. Three Japanese children with $\mathrm{X}$-linked alpha-thalassemia/mental retardation syndrome (ATR-X) [in Japanese]. No To Hattatsu 1998;30:283-89 
6. Gibbons RJ. ATR-X: a-thalassemia mental retardation-X-linked. In: Cassidy SB, Allanson JE, eds. Management of Genetic Syndromes. Hoboken, New Jersey: John Wiley \& Sons; 2010:97-109

7. Gibbons RJ, Wada T. ATRX and the X-linked a-thalassemia mental retardation syndrome. In: Epstein CJ, Erickson PP, Wynshaw-Boris A, eds. Inborn Errors of Development. 2nd ed. New York: Oxford University Press; 2008:943-54

8. Bérubé NG, Mangelsdorf M, Jagla M, et al. The chromatin-remodeling protein ATRX is critical for neuronal survival during corticogenesis. J Clin Invest 2005;115:258-67

9. Barkovich AJ, Raybaud C. Normal development of the neonatal and infant brain, skull, and spine. In: Barkovich AJ, Raybaud C. Pediatric
Neuroimaging. 5th ed. Philadelphia: Lippincott Williams \& Wilkins; 2012:20-80

10. Thienpont B, de Ravel T, Esch HV, et al. Partial duplications of the ATRX gene cause the ATR-X syndrome. Eur J Hum Genet 2007; 15:1094-97

11. Badens C, Lacoste C, Philip N, et al. Mutations in PHD-like domain of the ATRX gene correlate with severe psychomotor impairment and severe urogenital abnormalities in patients with ATRX syndrome. Clin Genet 2006;70:57-62

12. Kernohan KD, Jiang Y, Tremblay DC, et al. ATRX partners with cohesion and MeCP2 and contributes to developmental silencing of imprinted genes in the brain. Dev Cell 2010;18:191-202 